\title{
Study of aluminum nitride formation in plasma with an effect of double laser pulses on the D16T aluminum alloy in air
}

\author{
Trinh Ngoc Hoang ${ }^{1}$, Bazzal Khoder ${ }^{2}$, Fadaeian A.R. ${ }^{2}$, Zajogin A.P ${ }^{2}$ \\ ${ }^{1}$ Vinh University, Vinh City, Vietnam \\ ${ }^{2}$ Belarusian State University, Minsk, Belarus
}

Received August 27, 2016; accepted September 28, 2016; published September 30, 2016

\begin{abstract}
In this paper, we propose new opportunities to increase the proportion of various aluminum ions in near-surface laser plasma in the regimes of ablation of aluminum alloy surfaces by defocused double laser pulses, and AlN nanoclusters under the sequential influence of double pulses on the target (D16T aluminum alloy). The experimental results show that the intensity of spectral lines of $\mathrm{Al}$ ions reaches its maximum when time intervals between the double pulses of the order of $5 \div 15 \mu \mathrm{s}$, the pulse energy of $42 \mathrm{~mJ}$ (Al II) and $38 \mathrm{~mJ}$ (Al III). The percentage of AlN nanoclusters reaches a maximum at the energy of pulses of about $40 \mathrm{~mJ}$, defocus of $0 \mathrm{~mm}$ and the number of pulses of $25 \div 30$.
\end{abstract}

Currently, one of the materials in terms of its future prospects and applications in microelectronics and optoelectronics is aluminum nitride $(\mathrm{AlN})$, and ceramic materials based on it. The nitrides of metals have an unusual combination of properties. AlN has high thermal conductivity, comparable with the thermal conductivity of copper and silver (up to $260 \mathrm{~W} \cdot \mathrm{m}^{-1} \mathrm{~K}^{-1}$ ) at high values of electrical resistance (up to $10^{14} \Omega \cdot \mathrm{cm}$ ). Due to a large bandgap $(6.2 \mathrm{eV})$, AlN solid solutions can be used in semiconductor devices operated under extreme conditions of high temperature, high radiation and chemically aggressive influence. The possible applications include flame and temperature sensors, plasma diagnostics and detection of traces of rocket engines, etc.

At the present time, to obtain AlN in the form of powders or thin films and coatings, research groups begin to apply the methods of laser irradiation on $\mathrm{Al}$ in the atmosphere of activated nitrogen under pressure [1-3].

Laser ablation of solids with nanosecond pulses of moderate intensity is used in many scientific and practical applications. Pulsed laser deposition is widely used for forming thin-film structures and coatings from various materials. The physical processes associated with the formation of surface laser plasma, with its dispersion and deposition on the substrate is so multifactorial that one cannot provide enough simple laws describing these processes [4-5]. The method of pulsed laser deposition has certain inherent disadvantages, one of which is the formation of microdroplets $(0.1$ to $1 \mu \mathrm{m})$ at the ablation of targets [4-6]. In this connection, the problem of formation of structures is an urgent task, requiring modernization of

http://www.photonics.pl/PLP technological processes specific to their physicochemical properties.

The analysis and purposeful change of a component, charge and energy distributions of the laser torch is possible on the basis of an additional impact on the primary plasma of additional laser exposure. When using the schemes and methods of double-pulse laser exposure at different angles of incidence on the target and plasma, we can simultaneously perform high-sensitivity spectral analysis [4], [7-8], to monitor the concentration of excited and charged particles in the plasma and control plasma composition.

The purpose of this paper is to show the possibility and to determine the conditions of obtaining by the method of ablation a series of double laser pulses in aluminum targets in the air of AIN nanoclusters for their use in production technologies of nanocrystals and deposition of thin films.

For the research using a multichannel laser atomicemission spectrometer LSS-1, the excitation source is a double-pulse neodymium laser with adjustable energy and interval between pulses (model LS2131 DM). The laser can operate with a pulse repetition frequency up to $10 \mathrm{~Hz}$ at a wavelength of $1064 \mathrm{~nm}$. The pulse duration is approximately $15 \mathrm{~ns}$. The time delay between double pulses can vary from 0 to $100 \mu$ s in steps of $1 \mu$ s. Laser radiation focuses on the sample using an achromatic condenser with a focal length of $104 \mathrm{~mm}$.

In our earlier work [7] there were results of studies concerning the effect of the second pulse on near-surface processes in plasma and the dynamics of luminescence of atomic and ion lines of aluminum at various time intervals between laser pulses. From the data obtained, it was found that the line intensity of doubly charged ions Al III reaches a maximum at time intervals between two pulses of the order of $10 \div 15 \mu \mathrm{s}$, and for singly charged ions - in the range of $5 \div 10 \mu \mathrm{s}$. The optimal interval between pulses is $10 \mu \mathrm{s}$ and has been used in this work.

The dynamics of changes in atomic and ionic composition of near surface plasma and the formation of AlN nanoclusters in the depths of the crater were studied by multi-channel atomic emission spectrometry for the effect of double laser pulses on the plate of an aluminum alloy 
D16T with a thickness of $1 \mathrm{~mm}$ from their energy of $(15 \div 60 \mathrm{~mJ})$ and changing the power density by defocusing the exposing radiation.

The formation dynamics of one of the products of interaction of aluminum atoms with the nitrogen of AlN was studied by the emission spectra of this molecule when exposed to a series of single and double laser pulses on an aluminum target in air atmosphere. The most intense vibronic bands in the emission spectra are the bands with the wavelengths of 508.05 and $512.3 \mathrm{~nm}$.

Our study results showed that the highest band intensity is observed for the interval between pulses $6 \div 12 \mu$ s. Using the interval of $10 \mu \mathrm{s}$, we conducted a study of the formation process of AlN nanoclusters in dependence on pulse energy and the defocus. These results are shown in Fig. 1.
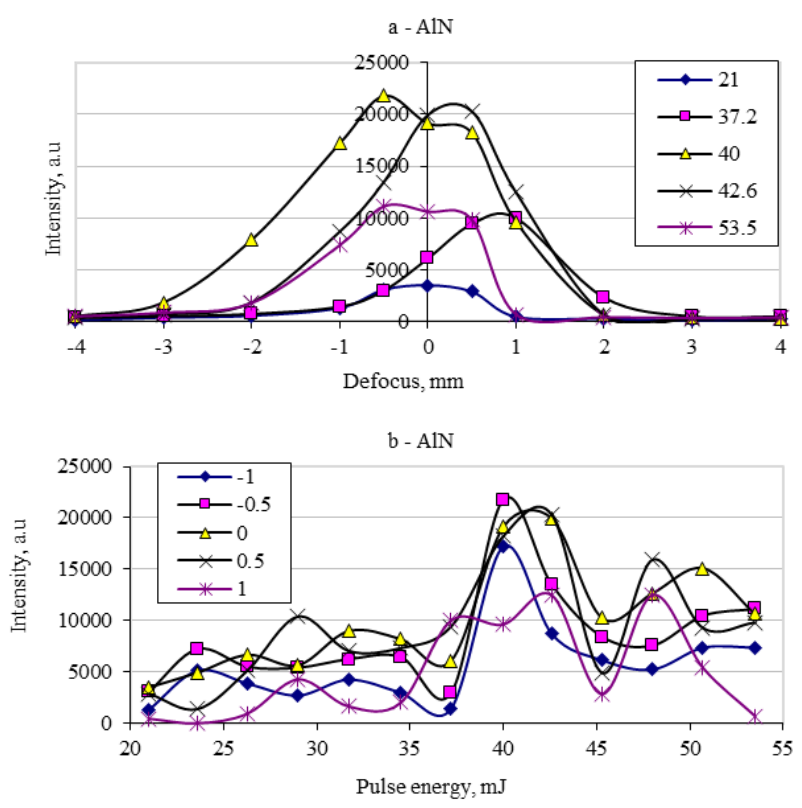

Fig. 1. The dependence of the band intensity of AlN $(512.3 \mathrm{~nm})$ in the spectra on defocus and pulse energy.

In the box: $\mathrm{a}$ - energy, $\mathrm{mJ} ; \mathrm{b}$ - the magnitude of defocus, $\mathrm{mm}$.

As can be seen from the obtained data, the formation of AlN nanoclusters increases at energy of about $40 \mathrm{~mJ}$ and defocus of about $0 \mathrm{~mm}$.

As it is well known, the most important role in the formation of ions and nanoclusters in laser plasma is the processes of ionization and recombination of ions [9-10]. The process of ionization is defined as the flux density of laser radiation and the ionization potential of atoms constituting the laser target and the diameter of the focusing spot [4].
Figure 2 shows the dependence of the intensity of Al II $(466.3 \mathrm{~nm})$ and $\mathrm{Al}$ III $(452.92 \mathrm{~nm})$ in the spectra on defocus and pulse energy.
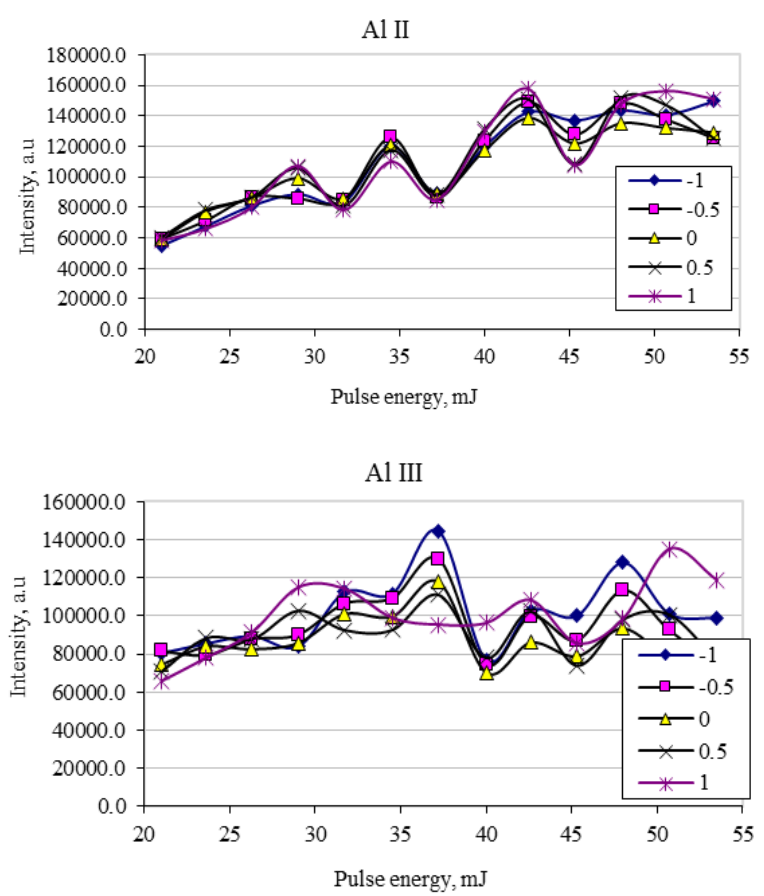

Fig. 2. The dependence of the intensity of $\mathrm{Al} \mathrm{II} \mathrm{(466.3nm)} \mathrm{and} \mathrm{Al} \mathrm{III}$ $(452.92 \mathrm{~nm})$ in the spectra on defocus and pulse energy (in the box: defocus, $\mathrm{mm}$ )

These results show a good correlation between the increase of Al II intensity and a significant decrease of the Al III intensity (at energies of more than $40 \mathrm{~mJ}$ ). This means that when the concentration of $\mathrm{Al}$ ions in the plasma increases, the concentration of $\mathrm{Al}$ III ions decreases. As it is well known, this phenomenon is related to the chemical interaction of $\mathrm{Al}$ ions in the plasma.

Using the above interval and energy, we conducted a study of the formation of AlN depending on the number of pulses (number of pulse pairs) impinging on the target. The results are shown in Fig. 3.

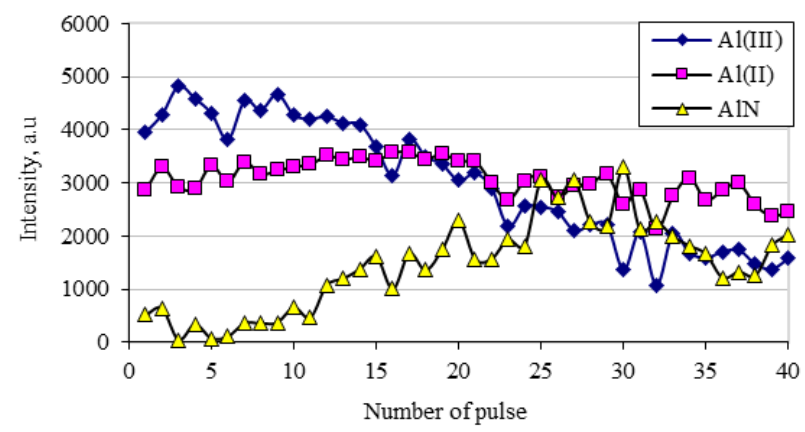

Fig. 3. The dependence of the band intensity of the AlN on the number of pulses impinging on the target 
As can be seen from the data in Fig. 3, the formation of AlN nanoclusters increases substantially with the number of pulses and the percentage of AlN nanoclusters reaches a maximum at the number of pulses of $25 \div 30$. The presence on the surface of aluminum oxide accelerates the beginning of the formation as radicals, $\mathrm{AlO}$ and $\mathrm{AlN}$.

It is known that the reactivity of oxygen at standard conditions is high and is associated with the peculiarities of the electronic structure of a molecule of $\mathrm{O}_{2}$. The oxygen molecule is a biradical, paramagnetic, the bond order is 2. Radicals have high reactivity, their chemical interaction occurs at low activation energy. Therefore, in reactions with aluminum, nitrogen has more advantage than oxygen, it is because the energy to break a chemical bond in a molecule of $\mathrm{O}_{2}$ is $493 \mathrm{~kJ} / \mathrm{mol}$, which is 2 times less than the energy of dissociation of molecule $\mathrm{N}_{2}$ $(940 \mathrm{~kJ} / \mathrm{mol})$, the order of which is equal to 3 [11].

However, in this case it should be noted that the molecule of oxygen under the action of electromagnetic radiation and high temperatures (the temperature inside the plasma of about $7000^{\circ} \mathrm{C}$ ) can pass from the paramagnetic triplet state $\mathrm{O}_{2}\left(\mathrm{X}^{3} \Sigma_{\mathrm{g}}^{-}\right)$into a singlet state $\mathrm{O}_{2}\left(\mathrm{a}^{1} \Delta_{\mathrm{g}}\right)$, that is, the formation of nitrides occurs when photochemical decontamination of oxygen transfers in a singlet (inactive) state, characterized by an increase in entropy of activation [12]. Thus, the emission of a hot sample, accompanying the combustion process, reduces the activity of oxygen, and the activated nitrogen reacts at a high temperature according to its reactivity. This is confirmed by the change in the concentration of nitrogen ions in plasma with defocusing, which is shown in Fig. 4.

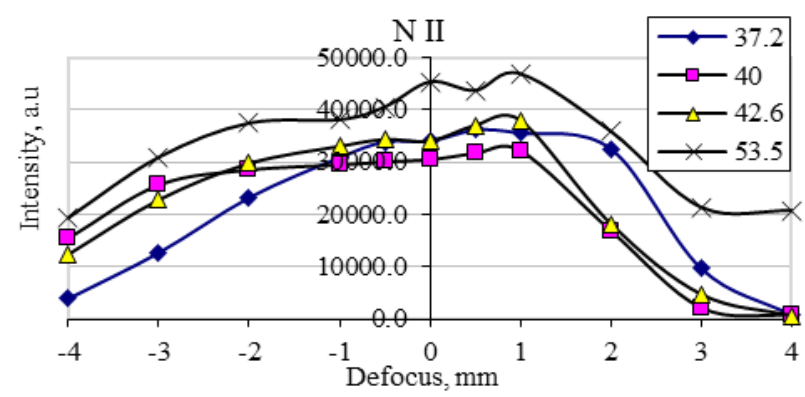

Fig. 4. The dependence of the intensity of ionic lines of nitrogen N II $(399.5 \mathrm{~nm})$ in the spectra on defocus and the pulse energy (in the box: energy, $\mathrm{mJ}$ )

As shown in Fig. 4, N II intensity, although weakly dependent on pulse energy, is strongly dependent on defocus, and reaches a maximum at a defocus of $0 \div 1 \mathrm{~mm}$.

In this work, we investigated the influence of experimental parameters on the formation of AlN nanoclusters. The experimental results show that the intensity of the spectral lines of $\mathrm{Al}$ ions reaches a maximum with the time intervals between double pulses of the order of $5 \div 15 \mu \mathrm{s}$, the energy of $42 \mathrm{~mJ}$ (Al II) and 38 $\mathrm{mJ}$ (Al III); The percentage of AlN nanoclusters reaches a maximum at the energy of pulses of about $40 \mathrm{~mJ}$, defocus of $0 \mathrm{~mm}$ and number of pulse of $25 \div 30$. Based on this, we can monitor and control the plasma characteristics.

Overall, the positive results of the experiments should be considered as a basis for further work on improvement of the process of the vacuum-free of laser deposition and optimization of the technological parameters. A significant expansion is also possible concerning deposited coatings including a mixed connection of hightemperature aluminum nitride - titanium nitride, which involves chemical interaction of evaporated particles of the target with the surrounding gas.

Our study shows the possibility of controlling plasma characteristics and the ability to obtain the necessary concentrations and the receipt of $\mathrm{AlO}$ and $\mathrm{AlN}$ radicals in plasma when the surface of oxidized aluminum is exposed to defocused consecutive double laser pulses.

\section{References}

[1] Zheng Biju, Hu Wen, J. Semicond. 37, 063003 (2016).

[2] J.A. Perez, I.P. Vera, H. Riascos, J.C. Caicedo, J. Physics: Conference Series 511, 1 (2014).

[3] D.-M. Marin, F. Stokker-Cheregi, M. Dumitru, V. Ion, M. Dinescu, Romanian Reports in Phys. 66, 1118 (2014).

[4] L.T. Sukhov, Laser spectral analysis (Novosibirsk 1990).

[5] T.V. Kononenko, V.I. Konov, E.N. Lubnin, F. Dausinger, Quantum Electr. 33, 189 (2003).

[6] V.K. Goncharov, K.V. Kozadaev, J. Eng. Physics 83, 80 (2010).

[7] A.R. Fadaiyan, N.H. Trinh, A.P. Zazhogin, Vestnik BSU 1, 27 (2011).

[8] A.R. Fadaian, A.P. Zazhogin, Vestnik BSU 1, 14 (2009).

[9] N.E. Kask, S.V. Michurin, G.M. Fedorov 33, 57 (2003).

[10] V.P. Krainov, M.B. Smirnov, Uspekhi Fizicheskikh Hauk 170, 969 (2000)

[11] A.P. Ilin, L.O. Root, J. Science Siberia 1, 91 (2011).

[12] G. Herzberg, Electronic spectra and structure of polyatomic molecules (Mir Press 1969). 